\title{
fegindo
}

Revista Legado de Arquitectura y Diseño

ISSN: 2007-3615

ISSN: 2448-749X

legado_fad@yahoo.com.mx

Universidad Autónoma del Estado de México

México

\section{ALUMNOS LATINOAMERICANOS EN LA ÉCOLE SPÉCIALE D'ARCHITECTURE (1900-1939)}

\section{Ávila-Gómez, Andrés}

ALUMNOS LATINOAMERICANOS EN LA ÉCOLE SPÉCIALE D'ARCHITECTURE (1900-1939)

Revista Legado de Arquitectura y Diseño, vol. 15, núm. 27, 2020

Universidad Autónoma del Estado de México, México

Disponible en: http://www.redalyc.org/articulo.oa?id=477963263005

Esta obra está bajo una Licencia Creative Commons Atribución-NoComercial-SinDerivar 4.0 Internacional. 


\title{
ALUMNOS LATINOAMERICANOS EN LA ÉCOLE SPÉCIALE D'ARCHITECTURE (1900-1939)
}

\author{
LATIN AMERICAN STUDENTS AT THE ÉCOLE \\ SPÉCIALE D’ARCHITECTURE (1900-1939) \\ Andrés Ávila-Gómez andresavigom@gmail.com \\ Université Paris 1 Panthéon-Sorbonne, Francia
} Revista Legado de Arquitectura y Diseño,
vol. 15, núm. 27, 2020

Universidad Autónoma del Estado de México, México

Recepción: 04 Septiembre 2019 Aprobación: 02 Diciembre 2019

Redalyc: http://www.redalyc.org/ articulo.oa?id $=477963263005$
Resumen: El presente texto plantea un primer estudio global sobre la presencia entre 1900 y 1939 de alumnos latinoamericanos en la École Spéciale d'Architecture (ESA), institución fundada en París, en 1865, para la enseñanza de la arquitectura.

Con el propósito de compilar la información básica sobre los jóvenes latinoamericanos inscritos en la École Spéciale d'Architecture durante las cuatro décadas señaladas, se ha analizado fundamentalmente la información disponible en dos tipos de documentos conservados en los archivos de la institución: se trata de los registros de inscripción, y de los folios de notas.

Para contextualizar dicha información se toma como apoyo la historiografía sobre la École Spéciale d'Architecture producida durante el último cuarto de siglo. Además de indagar acerca de las principales características de su modelo pedagógico, y en general, de las dinámicas que pudieron enmarcar este doble fenómeno de circulación de personas y de transferencia de conocimientos entre Francia y América Latina, se ha logrado establecer con precisión la identidad de los 124 alumnos provenientes de 16 países de América del Sur, de América Central y del Caribe inscritos entre 1900 y 1939: de ellos, un total de 51 obtuvieron finalmente el diploma otorgado por la institución, a quienes cumplían con todos los requisitos académicos exigidos.

Con los resultados de esta investigación se pretende aportar elementos inéditos que contribuyan a esclarecer, corregir o complementar las lagunas y los errores presentes en una historiografía latinoamericana que ha abordado tangencialmente el tema de las transferencias culturales derivadas de la educación recibida en Francia por un número significativo de jóvenes latinoamericanos durante los siglos XIX y XX.

Palabras clave: enseñanza de la arquitectura, método pedagógico, transferencia cultural. Abstract: This text presents a first global study on the presence between 1900 and 1939 of Latin American students at the École Spéciale d'Architecture (ESA), an institution founded in Paris, in 1865, for the teaching of architecture.

In order to compile the basic information on the young Latin Americans enrolled at the École Spéciale d'Architecture during the four decades mentioned above, we have mainly analysed the information available in two types of documents kept in the institution's archives: the registration records and the report cards.

To put this information into context, we have relied on the historiography of the Ecole Spéciale d'Architecture produced over the last quarter of a century. In addition to investigating the main characteristics of its pedagogical model and, in general, the dynamics that may have framed this dual phenomenon of the movement of people and the transfer of knowledge between France and Latin America, we have been able to establish precisely the identity of the 124 students from 16 countries in South and Central America and the Caribbean who were enrolled between 1900 and 1939.

The results of this research are intended to provide unprecedented elements that will help to clarify, correct or complement the gaps and errors present in a Latin American historiography that has tangentially addressed the issue of cultural transfers resulting from the education received in France by a significant number of young Latin Americans during the nineteenth and twentieth centuries. 
Keywords: architecture education, teaching method, cultural transfers.

\section{INTRODUCCIÓN}

En el distrito 14 de París, en el costado occidental del boulevard Raspail -justo enfrente del edificio de la Fondation Cartier, diseñado por Jean Nouvel- funciona desde 1904 la École Spéciale d'Architecture (ESA) (figura 1), fundada en 1864, bajo el nombre de École Centrale d'Architecture, y reconocida como establecimiento de utilidad pública en 1870 .

A pesar de la importancia de esta institución -que en 1869 cambió su nombre de "Centrale" por "Spéciale"- en el panorama de la enseñanza de la arquitectura en Francia durante los siglos XIX y XX, a partir de la década de 1990 se produjeron los primeros estudios históricos acerca de la ESA. Gracias a las investigaciones realizadas por el arquitecto e historiador de la arquitectura Frédéric Seitz, apareció publicado en 1995 un primer estudio sobre el periodo, que va desde la fundación de la ESA hasta el periodo de entreguerras (figura 2), es decir, durante la época en la cual la institución fue dirigida por Émile Trélat y más tarde por su hijo Gaston Trélat (figura 3).

En dicho contexto, en el cual la historia y el reconocimiento de la ESA han permanecido un poco a la sombra de la célebre École Nationale Supérieure des Beaux-Arts (ENSBA), el presente texto propone un primer análisis global acerca de la presencia entre 1900 y 1939 de alumnos latinoamericanos en esta institución parisina.

Para tal fin se han analizado documentos que exponen la identidad de aquellos alumnos, contextualizándolos y aportando elementos historiográficos que contribuyen a esclarecer el entorno y las dinámicas que enmarcaron esta modalidad de circulación de personas y su posterior incidencia en la transferencia de conocimientos.

Para ello, y ante la inexistencia de traducciones al español de los estudios y publicaciones de investigadores como Frédéric Seitz, la primera parte del artículo expone una síntesis acerca de la historia de la ESA y de las principales características de su modelo pedagógico, con el fin de entender de manera general los contenidos curriculares que moldearon la formación de dichos alumnos.

Se presenta de esta manera un conjunto de individuos inscritos en la ESA, independientemente de la trayectoria profesional desarrollada posteriormente: se encuentran por supuesto una serie de nombres plenamente reconocidos por las diferentes historiografías nacionales, tal y como sucede con los chilenos Alberto Cruz Montt y Ricardo Larraín Bravo; el boliviano Adán Sánchez; el portorriqueño Alfredo Wiechers Pieretti; el argentino Estanislao Pirovano, el venezolano Luis Malaussena; o el dominicano José Antonio Caro Álvarez.

Además de constituir una base con información precisa para estudios posteriores, el presente artículo pretende trazar un panorama general que permita esclarecer, corregir o complementar las lagunas y los errores presentes en una historiografía latinoamericana, que hasta ahora, 
ha abordado tangencialmente el tema de las transferencias culturales derivadas de la educación recibida en Francia por un número significativo de jóvenes latinoamericanos que al regresar a sus países de origen, influyeron profundamente tanto en la práctica como en la enseñanza de la arquitectura y el urbanismo.

\section{METODOLOGÍA}

A partir de la historiografía -aun escasa- sobre la ESA, y apoyándonos principalmente en las fuentes primarias disponibles en los archivos de la institución, se propone un balance cuantitativo del caso de los estudiantes latinoamericanos. Se ha dado prioridad al análisis de la información recogida en dos tipos de documentos consultados minuciosamente en los archivos de la École Spéciale d'Architecture: ${ }^{[1]}$ los registros de inscripción y los folios de notas. Igualmente, se han cotejado tales documentos con los listados históricos publicados en los años 1990 en los anuarios de la Société des Architectes Diplômés de l'École Spéciale d'Architecture SADESA (figura 4). A partir de este corpus se han construido una serie de tablas que permitirán a otros investigadores iniciar nuevas pesquisas o simplemente desarrollar otras lecturas interpretativas.

Es importante señalar que los resultados mostrados en el presente texto son parte de una investigación más amplia en la cual hemos abordado cuestiones similares en torno a la formación de alumnos latinoamericanos en la École Nationale Supérieure des Beaux-Arts (ENSBA), en la École Spéciale d'Architecture (ESA), y en el Institut d'Urbanisme de l'Université de Paris (IUUP). ${ }^{[2]}$

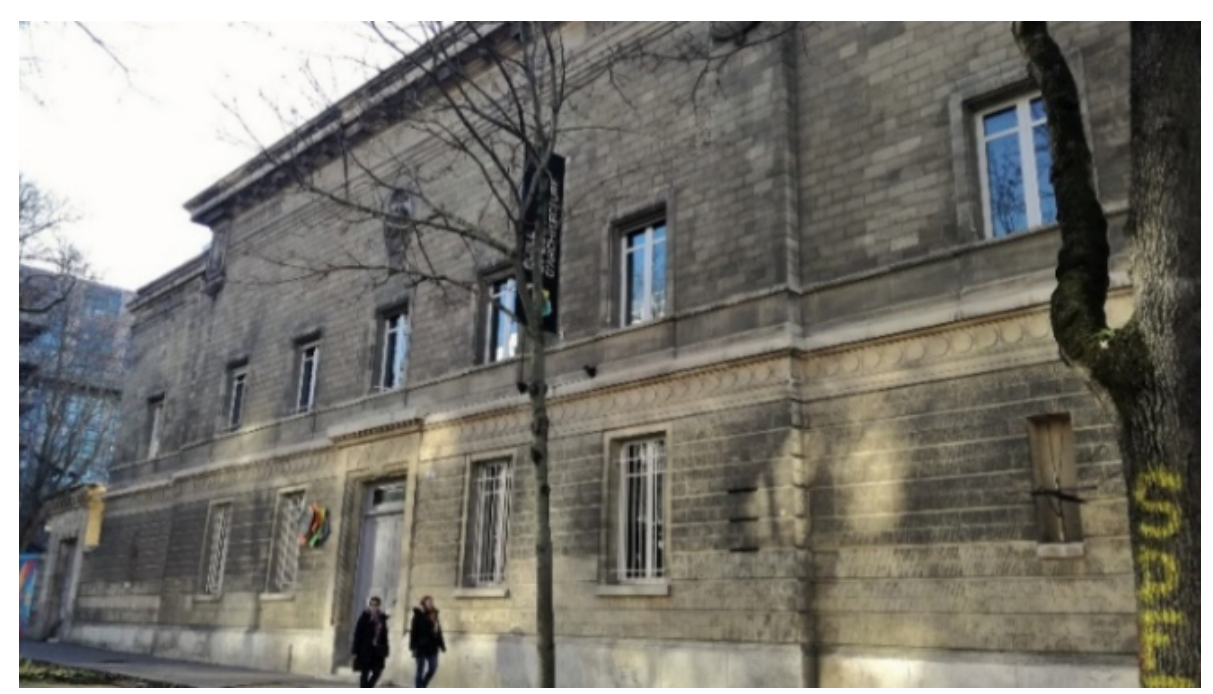

Figura 1. Vista del acceso principal a la ESA sobre el boulevard Raspail.

Fuente: Fotografía de Andrés Ávila Gómez, diciembre 2019. 


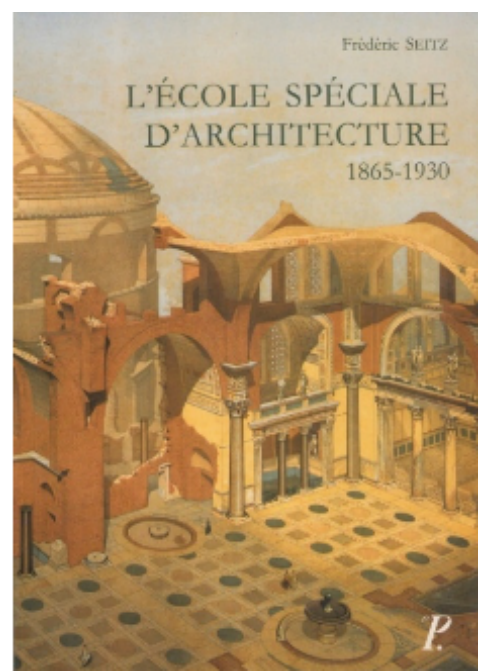

Figura 2. Portada del libro escrito por Frédéric Seitz sobre la historia de la ESA. Fuente: Picard (1995).

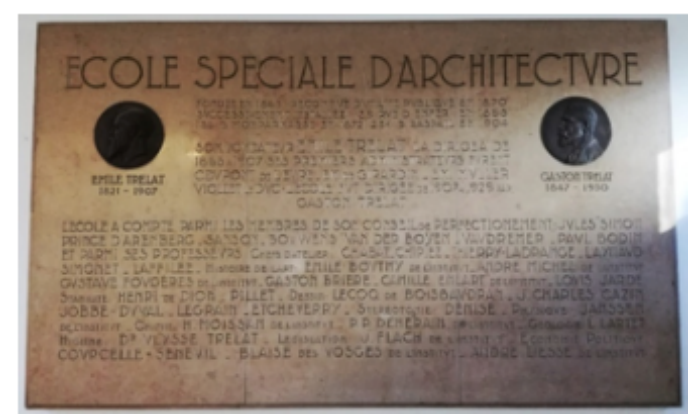

Figura 3. Placa conmemorativa en el hall de entrada de la ESA: se aprecian en la parte superior dos relieves representando el perfil de Émile Trélat (izquierda) y de Gaston Trélat (derecha). Fuente: Fotografía de Andrés Ávila Gómez, diciembre 2019.

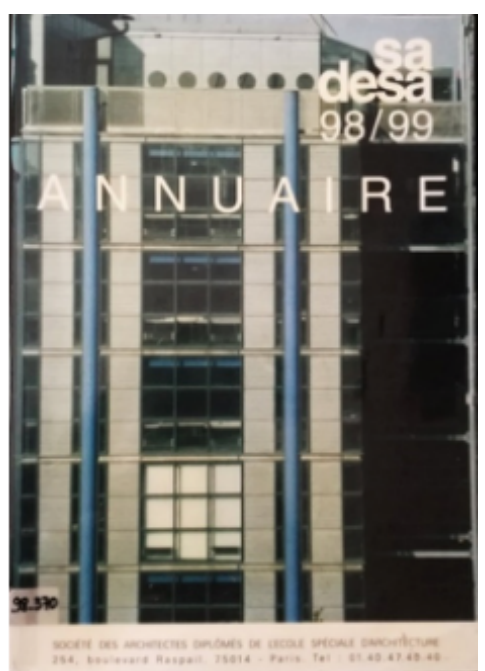

Figura 4. Cubierta del anuario 1998-1999 de la Société des Architectes Diplômés de l'École Spéciale d'Architecture - SADESA. Fuente: Archives Bibliothèque ESA. 


\section{UNA NUEVA ESCUELA FRENTE A LA HEGEMONÍA BEAUXARTIANA}

La aparición de la École Centrale d'Architecture en la segunda mitad del siglo XIX constituye un momento clave en la renovación de la formación de los arquitectos en Francia. Para ello, además del compromiso irrestricto de su primer director Émile Trélat (1821-1907), diplomado de la École Centrale des Arts et Manufactures y profesor de construcciones civiles en el Conservatoire des Arts et Métiers, fue vital el apoyo dado a la joven institución por personalidades del campo de la arquitectura que de diferentes maneras promovieron su consolidación como alternativa al academicismo de la ENSBA. ${ }^{[3]}$

En sus orígenes, las sesiones inaugurales anuales contaban con la presencia de algún invitado especial, entre los cuales figuraban arquitectos tan prestigiosos como César Daly, Gabriel Davioud, Héctor Horeau, Henri Labrouste, Constant Moyaux, Charles Thierry, Joseph Vaudremer, o William Bouwens van der Boyen (Seitz, 1995: 107).

Citando algunos textos del propio Trélat, ${ }^{[4]}$ el arquitecto e historiador, Jacques Lucan, resume así la esencia de lo que la ESA buscaba difundir (Lucan, 2017: 105):

Opposé à toute "théorie du beau invariable, du beau circonscrit dans les types, du beau trouvé une fois pour toutes dans les contrées privilégiées, du beau né en Grèce, réédité à Rome", Trélat cherche à développer un enseignement plus rationnel, à l'image de celui donné aux ingénieurs civils, mais sans pour autant abandonner le système des ateliers: "L'atelier, on doit le répéter, est ainsi le centre autour duquel gravite tout l'enseignement. [5]

Sin embargo, las transformaciones promovidas tras la sucesión de Émile, en la cabeza de su hijo Gaston Trélat (1847-1929), quien fungió como director entre 1907 y 1929, son descritas por Seitz como un periodo obscuro para la ESA, por cuanto estas supusieron una especie de "retorno al pasado", que mantuvo a la institución casi al margen del movimiento reformador de la arquitectura que tuvo lugar en Francia en los años 1920. Y aunque la gran mayoría de arquitectos diplomados de la ESA ocupaban cargos tradicionales sin mayor figuración, algunos desarrollaron brillantes carreras en Francia y Europa, entre ellos: Robert Mallet-Stevens (1886-1945), Henri Prost (1874-1959), Jean Walter (1883-1957), Jean Badovici (1893-1956), Adrienne Gorska (1899-1969), Jean Ginsberg (1905-1983) y Pierre Vago (1910-2002). ${ }^{[6]}$

En un artículo publicado en 1925 en el número 23 de la revista $L$ 'Esprit Nouveau, se hacía eco de la situación de la ESA:

\footnotetext{
Et voilà ce qui est arrivé ! Ce qui devait arriver! Un monsieur qui est architecte et père de l'un des élèves de Mallet-Stevens, a surgi devant M. Trélat, le Directeur de l'École. L'architecte a brandi son parapluie, il a hurlé : «Vous vous f... de moi; vous voulez tuer mon fils. Vous l'empoisonnez, vous l'encanaillez! Il n'aura jamais de commande [...] M. le Directeur de l'École qui n'était déjà pas très fixé et tremblait par anticipation, s'est mis à trembler pour de bon. Il a attrapé Mallet-Stevens, lui a dit que ça ne pouvait plus durer, qu'il fallait changer ça, revenir aux traditions de son honorable école. Cré Dieu! M. Trélat lui, il y avait fait une carrière (et de
} 
modestie encore). Mallet-Stevens qui est propre et un peu fier s'en est allé. Les jeunes gens enthousiastes finiront leurs études dans Vignole et le Grand Palais [...]. [7]

Este episodio da cuenta del descontento que generaban algunos aspectos y prácticas presentes en el seno de la ESA, criticadas desde afuera y denunciadas por ser consideradas como sinónimo de un anquilosamiento que iba en contra de los ideales que habían conducido originalmente a su fundación. Además, tales controversias estaban ligadas al debate disciplinar en torno a las tres tendencias que se confrontaban desde el último tercio del siglo XIX: por un lado, se encontraban quienes como Viollet-le-Duc ${ }^{[8]}$ profesaban una ideología carismática del arquitecto-artista que rechazaba, tanto la reglamentación del título como del ejercicio mismo de la profesión: «onne "diplôme" pas un artiste et on ne réglemente pas sa "vocation" [...]». Por el otro, estaban quienes deseaban que tanto el título como el ejercicio de la profesión fuesen reglamentados, con el propósito de asegurar a los arquitectos diplomados el monopolio efectivo de la actividad. Por último, estaban quienes pedían la protección por ley del título de arquitecto DPLG, ${ }^{[9]}$ es decir, el reconocimiento jurídico de una competencia específica sin reivindicar la exclusividad del ejercicio de la profesión (Moulin et al., 1973: 29).

Finalmente, con la ley del 31 de diciembre de 1940, validada por el decreto del 18 de octubre de 1945, se instauró la Ordre des architectes para asegurar la protección del título: «nulnepeut s'intituler architecte s'iln'est pas diplômé par l'une des écoles reconnues à cette fin par l'État»; siendo estas:

1. École Nationale Supérieure des Beaux-Arts (y sus satélites regionales)

2. École Spéciale d'Architecture

3. École Nationale d'Ingénieursde Strasbourg

\section{LA FORMACIÓN Y LOS ATELIERS}

El sistema de enseñanza puesto en marcha por Émile Trélat se inspiró esencialmente en el programa impartido entonces en la École Centrale des Arts et Manufactures, a lo cual sumó una serie de innovaciones que le hizo sobresalir desde entonces dentro de un panorama de la enseñanza de la arquitectura que gravitaba en torno al modelo de la ENSBA. ${ }^{[10]}$

La formación en el atelier constituía el punto de encuentro de todas las asignaturas impartidas en la ESA: en el anfiteatro, el alumno aprendía a "entender", y en el atelier aprendía a "amar". Según la doctrina apreciada por Trélat, a través de los ejercicios propuestos en las clases de anfiteatro y en el atelier, el alumno descubría progresivamente el mundo de las formas para luego aprender a crear por sí mismo los volúmenes y los espacios resolviendo armoniosamente el conflicto entre la luz y los materiales (Seitz, 1995: 89).

Un folleto que data de $1867^{[11]}$ precisa las condiciones de trabajo en un atelier de la escuela: 
C'est à l'atelier que se font les travaux fondamentaux qui constituent l'exercice permanent de l'architecture et qui éveillent l'artiste par une lente assimilation des procédés d'expression réservés à l'architecte ; c'est là que se poursuivent les études graduées, qui prennent les noms de copies, relevés, projets (esquisses ou rendus), concours, etc., suivant le degré d'avancement de l'élève. A l'atelier, l'élève est libre; il distribue et utilise son temps comme il l'entend pour satisfaire aux exigences variées de l'enseignement; mais il profite à sa volonté des conseils régulièrement offerts par le chef d'atelier, et il use, à ses heures, des documents, qu'il trouve réunis à la bibliothèque de l'établissement. ${ }^{[12]}$

El diploma de la ESA constituyó un elemento precursor en el medio francés, por cuanto fue estipulado desde 1864 por los fundadores de la escuela, oficializado por el Conseil d'administration en diciembre de 1867, y otorgado desde la primera promoción de alumnos. La ENSBA por su parte creó su diploma en noviembre de 1867.

Ya entrado el siglo XX y tras una serie de reformas durante los años de Gaston Trélat, los alumnos de la ESA tenían cada año la opción de cambiar de atelier; y para poder obtener el diploma conferido por la institución, debían presentar tres concursos, validando igual número de certificados denominados así:

- Concours de Technique: Certificat d'architecte technicien ${ }^{[13]}$

- Concours de Plastique: Certificat d'architecte plasticien ${ }^{[14]}$

- Concours de Salubrité: Certificat d'architecte hygiéniste ${ }^{[15]}$

Con la llegada en 1929 de Henri Prost ${ }^{[16]}$ (1874-1959) a la dirección de la ESA - cargo que ocupó hasta su muerte en 1959-, el programa de estudios sufrió algunas modificaciones importantes, entre las cuales, la extensión del tiempo de estudios. Por otro lado, en aquel momento se invitó a Auguste Perret (1874-1954), una figura de primer nivel de la arquitectura francesa y europea, para desempeñar el rol de chef d'atelier, ${ }^{[17]}$ con lo cual se propició el espacio para que surgiera en el seno de la ESA toda una generación de brillantes alumnos.

En dicho contexto, y ante la calidad de la formación en arquitectura impartida en estas dos escuelas, una nueva tendencia se hizo presente: los alumnos diplomados de la ESA y la ENSAD ya no vieron como algo necesario continuar sus estudios inscribiéndose en la ENSBA, tal y como hacían los alumnos diplomados a principios de siglo. ${ }^{[18]}$ En otras palabras, como lo señala Dumont: «On ne pouvait donc plus tenir ces deux institutions pour des «petites écoles» [...]» (1989: 66).

Hoy en día, los folios de notas nos permiten en su gran mayoría conocer el historial del desempeño académico de cada estudiante: estos presentan las notas con las cuales aprobaban o reprobaban las asignaturas seguidas durante los años cursados, organizados fundamentalmente en tres años denominados $3 e$ classe (primer año), 2e classe (segundo año), y le classe (último año); compuestos cada uno a su vez de tres trimestres. Tal y como aparece en dichos folios, era ofrecida una gama de asignaturas incluyendo algunas electivas en diferentes especialidades. Así, por ejemplo, encontramos un total de 11 asignaturas ofrecidas en la $3 e$ classe, y 14 asignaturas en la $2 e$ classe (tabla 1 ), muchas de las 
cuales resultarían totalmente extrañas hoy en una escuela de arquitectura: tal es el caso de Geología, Química aplicada, Economía política, o Contabilidad.

El folio de cada estudiante señala también, según el caso, si el alumno obtuvo algún premio o medalla, dentro del abanico de opciones en las cuales podían participar a lo largo de su escolaridad; así como la posición ocupada por el estudiante dentro de la classe.

Tabla 1. Asignaturas ofrecidas en la ESA durante el periodo de entreguerras.

\begin{tabular}{|l|l|l|}
\hline & $2^{\circ}$ classe & $3^{\circ}$ classe \\
\hline 1 & Architecture & Architecture \\
\hline 2 & Dessin d'imitation & Dessin d'imitation \\
\hline 3 & Stabilité des constructions & Géométrie descriptive \\
\hline 4 & Emploi du métal & Construction \\
\hline 5 & Physique appliquée & Mathématiques générales \\
\hline 6 & Chimie appliquée & Ombres \\
\hline 7 & Electricité appliquée & Stéréotomie (épurés) \\
\hline 8 & Géologie & Géologie \\
\hline 9 & Topographie & Topographie \\
\hline 10 & Economie politique & Perspectives \\
\hline 11 & Législation & Travaux de vacances \\
\hline 12 & Comptabilité & \\
\hline 13 & Machinerie & \\
\hline 14 & Travaux de vacances & \\
\hline
\end{tabular}

Fuente: Elaboración propia (Archives Bibliothèque ESA).

\section{LATINOAMERICANOS DIPLOMADOS Y NO DIPLOMADOS DE LA ESA}

El caso de los estudiantes latinoamericanos inscritos en la ESA, ya sea que hayan obtenido el diploma o no, nunca ha sido abordado hasta ahora. $\mathrm{Y}$, sin embargo, resulta fundamental estudiar este caso de circulación de estudiantes, ya que, desde la fundación de esta escuela francesa de arquitectura, se hicieron presentes alumnos provenientes de las por entonces jóvenes naciones latinoamericanas: basta mencionar el caso de Jules-Émile Jéquier nacido en Santiago de Chile en 1866 (hijo de Henri Jéquier, ${ }^{[19]}$ un ingeniero francés egresado de la École Centrale des Arts et Manufactures), quien obtuvo su diploma de la ESA en 1886.

El estudio minucioso de los registros de inscripción, y de los folios de notas de la ESA nos ha permitido confirmar la presencia entre 1900 y 1939, de 124 alumnos de origen latinoamericano (tablas 2 y 3), de los cuales 51 obtuvieron el diploma (tabla 4) que la escuela concedía a quienes cumplían con la totalidad de los requisitos académicos. Además de los datos sobre alumnos provenientes de países de América del Sur y de América Central, se han incluido también los datos de aquellos jóvenes nacidos en la región del Caribe, concretamente en cuatro países: Cuba, Haití, República Dominicana y Puerto Rico. Se constata que fueron en total 16 países de los cuales provenían estos alumnos, dos no corresponden a países hispanófonos: Brasil, que es lusófono; y Haití, que es francófono. 
Tabla 2. Estudiantes latinoamericanos inscritos (diplomados y no diplomados) en los registros de la ESA entre 1900 y 1939, según nacionalidades.

\begin{tabular}{|l|l|l|l|}
\hline & Nacionalidad & $N^{\circ}$ estudiantes & Porcentaje \\
\hline 1 & Argentina & 37 & $30 \%$ \\
\hline 2 & Brasil & 13 & $11 \%$ \\
\hline 3 & México & 12 & $9,5 \%$ \\
\hline 4 & Chile & 12 & $9,5 \%$ \\
\hline 5 & Haití & 12 & $9,5 \%$ \\
\hline 6 & Colombia & 8 & $7 \%$ \\
\hline 7 & Bolivia & 6 & $5 \%$ \\
\hline 8 & Perú & 6 & $5 \%$ \\
\hline 9 & Venezuela & 4 & $3 \%$ \\
\hline 10 & R. Dominicana & 2 & $1,5 \%$ \\
\hline 11 & Costa Rica & 2 & $1,5 \%$ \\
\hline 12 & Cuba & 2 & $1,5 \%$ \\
\hline 13 & Ecuador & 2 & $1,5 \%$ \\
\hline 14 & Paraguay & 2 & $1,5 \%$ \\
\hline 15 & Puerto Rico & 2 & $1,5 \%$ \\
\hline 16 & Uruguay & 2 & $1,5 \%$ \\
\hline & ToTAL & $\mathbf{1 2 4}$ & $\mathbf{( 1 0 0 \% )}$ \\
\hline
\end{tabular}

Fuente: Elaboración propia (Archives Bibliothèque ESA).

Nuestra pesquisa incluye los tres primeros alumnos diplomados durante el cambio de siglo: se trata de los chilenos Alberto Cruz Montt (1879-1955), Miguel Ángel de la Cruz (1874-¿?) y Ricardo Larraín Bravo (1879-1945).

La revisión de los registros de inscripción y de los folios de notas exigió un ejercicio adicional de verificación debido a un inconveniente puntual: la francofonización (francisation) de los nombres. Debido a esta práctica habitual en la administración francesa de la época, se encuentran numerosos casos en los cuales el nombre de un alumno está escrito de manera diferente a como aparece en sus documentos de identidad. Así, encontramos, por ejemplo, a Michel Ange de la Cruz (Miguel Angel); a Adam Sánchez (Adán); a Jules Marie y a Charles Malbranche (Julio María y Carlos), a Thomas y Georges Saubidet (Tomás y Jorge); y a Ange Ceppi (Ángel), entre otros.

En 1911, el bonaerense Alberto Manuel Blancas obtiene su diploma, siendo el primero de los 18 argentinos graduados entre 1900 y 1939 , entre los 37 inscritos, constituyendo el principal grupo latinoamericano presente en la ESA, triplicando en todo caso el número de inscritos de las 4 nacionalidades que siguen en la lista: Brasil con 13 inscritos (5 diplomados), y luego Chile, México y Haití cada una con 12 inscritos (con 7, 4, y 3 diplomados respectivamente). Y aunque aparecen inscritos dos estudiantes de nacionalidad ecuatoriana (en 1921 y 1926), éste es el único país que no tuvo ningún alumno diplomado.

Sobresale también otro aspecto en cuanto al lugar de nacimiento de estos alumnos: 86 de ellos, es decir, el $70 \%$ de los latinoamericanos inscritos provenían de alguna capital nacional como Buenos Aires, Santiago, o Río de Janeiro; y el otro 30\% figura en los registros como proveniente de pequeñas ciudades (por ejemplo, los colombianos provenientes de Popayán, Riosucio y Subachoque). En el caso excepcional 
del boliviano Luis Iturralde Levy, inscrito en 1925 y diplomado en 1929, el registro indica como lugar de nacimiento la pequeña comuna de Perreux, ubicada en la Región parisina; lo cual, sin embargo, parece corresponder a un error en la transcripción del registro correspondiente.

En muchos casos, el paso por la ESA fue visto como una etapa importante de preparación en su intento por acceder posteriormente a la ENSBA, tal y como era costumbre entre los jóvenes alumnos europeos. Un ejemplo de esta dinámica lo constituye el caso del ya mencionado Jules-Émile Jéquier, estudiante chileno que tras obtener en 1886 su diploma de la ESA, ingresó al año siguiente a la ENSBA, ${ }^{[20]}$ tras ser admitido en $2 e$ classe en el atelier de Paul Blondel, ${ }^{[21]}$ obteniendo así su segundo diploma en 1889.

Así, al revisar los registros de la ESA correspondientes al periodo que concierne al presente estudio, se encuentran numerosos casos de alumnos que desertaban incluso antes de finalizar el primer año -generalmente con bajos resultados académicos-, algunos de los cuales se inscribían en otras instituciones parisinas en programas también relacionados con la arquitectura o el urbanismo: tal es el caso del ecuatoriano Cristóbal Pallares, quien desertó de la ESA, pero obtuvo en 1927 el diploma del IUUP; del colombiano Carlos Martínez Jiménez, quien se retiró de la ESA durante el primer año de estudios (figuras 5, 6, 7), pero consiguió diplomarse en 1930 del IUUP; o del también colombiano Manuel Sánchez, quien se diplomó únicamente del IUUP, en 1931. ${ }^{[22]}$

Debido a la exigencia académica de tales programas, y en la mayoría de los casos a causa también de un débil dominio de la lengua francesa, son realmente excepcionales los casos en los cuales los alumnos latinoamericanos se diplomaron en dos instituciones de este tipo: esto sucedió con el venezolano Ernesto Enrique Paradas (diploma IUUP en 1929, y diploma ESA en 1930), del uruguayo Norberto Palacios (diploma IUUP en 1930, y diploma ESA en 1931), y del argentino Luis Pérez Quesada (diploma IUUP en 1930, y diploma ESA en 1933).

Otros cuantos aparecen inscritos en varias instituciones aunque no obtuvieron finalmente el diploma en ninguna de ellas: tal es el caso del argentino Ernesto Emilio Lemos (1896- ¿?) inscrito en la ESA en 1915, y presente en el atelier de Henri Deglane en la ENSBA en 1919. ${ }^{[23]}$

Los datos recogidos en las tablas 3 y 5 muestran cómo, tras la turbulenta época de la Primera Guerra (únicamente 18 diplomados en la ESA entre 1915 y 1918, de los cuales un solo latinoamericano en 1916: el brasilero Octavio de Gouvea-Freire), a partir de los años 1920 se reactiva la llegada de alumnos latinoamericanos. 


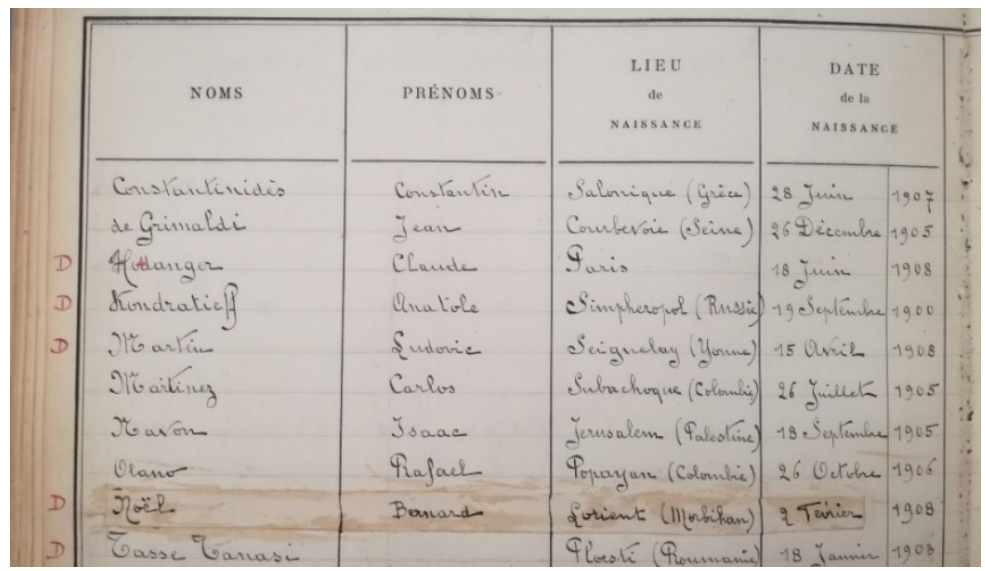

Figura 5. Extracto del registro de alumnos de la ESA en el cual

figuran inscritos los colombianos Carlos Martínez y Rafael Olano.

Fuente: Fotografía de Andrés Avila Gómez, junio 2019 (Archives Bibliothèque ESA).

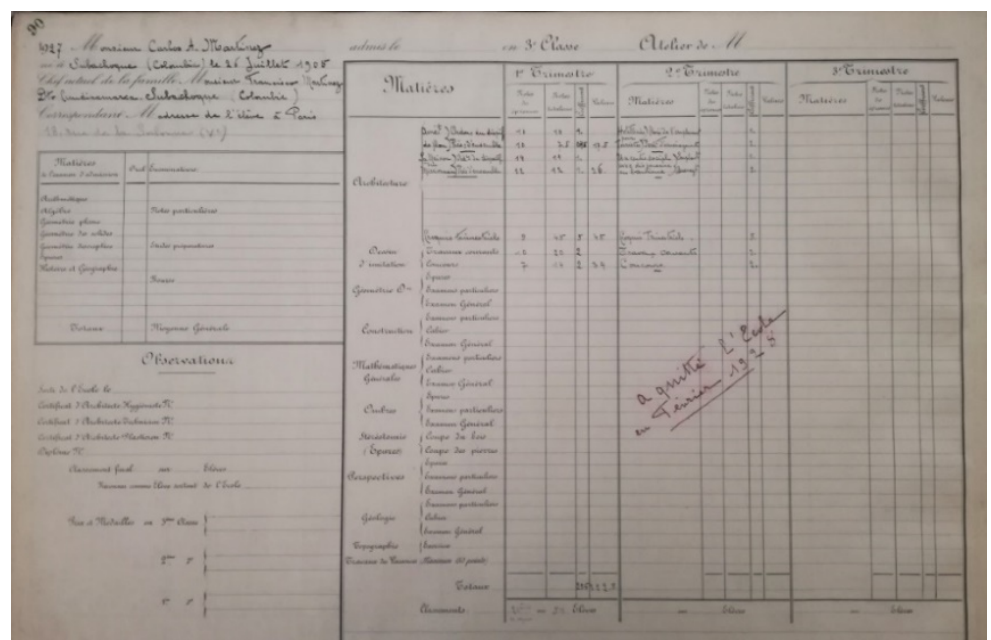

Figura 6. Primera página de notas del estudiante colombiano Carlos Martínez Jiménez, señalando su retiro de la ESA en febrero de 1928.

Fuente: Fotografía de Andrés Avila Gómez, junio 2019 (Archives Bibliothèque ESA).

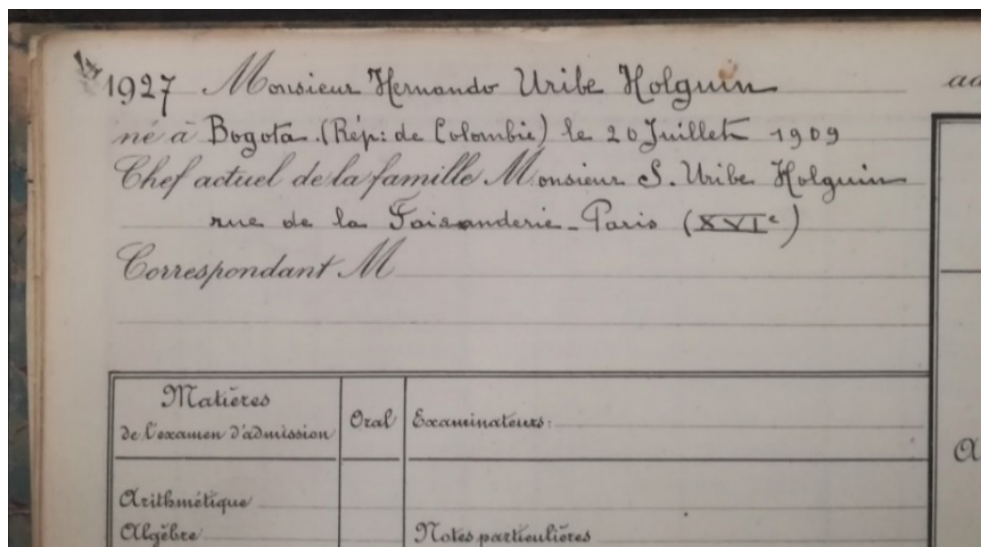

Figura 7. Detalle del encabezado en la primera página de notas del colombiano Hernando Uribe Holguín, inscrito en 1927 en la ESA, el mismo año que los también colombianos Carlos Martínez Jiménez y Rafael Olano. Fuente: Fotografía de Andrés Avila Gómez, junio 2019 (Archives Bibliothèque ESA). 
Tabla 3. Datos básicos de los estudiantes latinoamericanos inscritos en los registros de la ESA entre 1900 y 1939.

\begin{tabular}{|c|c|c|c|c|c|c|}
\hline & INSC. & \begin{tabular}{|l} 
NOMBRE \\
ALUMNO
\end{tabular} & \begin{tabular}{|l} 
LUGAR \\
NACIMIENTO
\end{tabular} & $\begin{array}{l}\text { FECHA } \\
\text { NACIM. }\end{array}$ & \begin{tabular}{|l} 
EDAD \\
INGR.
\end{tabular} & DIPLOMA \\
\hline \multicolumn{7}{|c|}{ Inscritos entre 1895 y 1909} \\
\hline 1 & 1896 & $\begin{array}{l}\text { Albert Cruz } \\
\text { Montt }\end{array}$ & \begin{tabular}{|l|}
$\begin{array}{l}\text { Valparaíso } \\
\text { (Chile) }\end{array}$ \\
\end{tabular} & 04-09-1879 & 17 & Si : 1899 \\
\hline 2 & 1897 & \begin{tabular}{|l} 
Michel \\
Ange de la \\
Cruz
\end{tabular} & $\begin{array}{l}\text { Santiago de } \\
\text { Chile }\end{array}$ & $16-07-1874$ & 23 & Si : 1900 \\
\hline 3 & 1897 & $\begin{array}{l}\text { Ricardo } \\
\text { Larraín } \\
\text { Bravo } \\
\end{array}$ & $\begin{array}{l}\text { Valparaíso } \\
\text { (Chile) }\end{array}$ & $09-04-1879$ & 18 & Si : 1900 \\
\hline 4 & 1901 & \begin{tabular}{|l|} 
Adam \\
Sánchez
\end{tabular} & La Paz & $06-12-1880$ & 21 & Si : 1904 \\
\hline 5 & 1902 & \begin{tabular}{|l|} 
Alfred \\
Wiechers
\end{tabular} & $\begin{array}{l}\text { Ponce (Puerto } \\
\text { Rico) }\end{array}$ & $26-03-1881$ & 21 & Si : 1905 \\
\hline 6 & 1903 & $\begin{array}{l}\text { Fernando } \\
\text { Ancona } \\
\text { Camara }\end{array}$ & $\begin{array}{l}\text { Mérida } \\
\text { (México) }\end{array}$ & $27-02-1885$ & 18 & No \\
\hline 7 & 1904 & $\begin{array}{l}\text { Jules Marie } \\
\text { Malbranche }\end{array}$ & Buenos Aires & $23-09-1886$ & 18 & No \\
\hline $8 a$ & 1905 & \begin{tabular}{|l|} 
Ernest \\
Chaline
\end{tabular} & $\begin{array}{l}\begin{array}{l}\text { Pernambuco } \\
\text { (Brasil) }\end{array} \\
\text { (Bris }\end{array}$ & $02-06-1888$ & - & (si) \\
\hline $9 a$ & 1905 & \begin{tabular}{|l|} 
Ernesto \\
Morelos
\end{tabular} & $\begin{array}{l}\text { Guadalajara } \\
\text { (México) }\end{array}$ & 04-08-1882 & - & (si) \\
\hline 10 & 1905 & $\begin{array}{l}\text { Guillermo } \\
\text { Rey y Boza }\end{array}$ & Lima & $15-05-1885$ & 20 & No \\
\hline 11 & 1905 & $\begin{array}{l}\text { Georges } \\
\text { Lauture }\end{array}$ & Jacmel (Haití) & $13-10-1887$ & 18 & No \\
\hline 12 & 1906 & Ange Ceppi & $\begin{array}{l}\text { Santiago de } \\
\text { Chile }\end{array}$ & $27-09-1886$ & 20 & Si : 1908 \\
\hline $8 b$ & 1906 & \begin{tabular}{|l|} 
Ernest \\
Chaline
\end{tabular} & $\begin{array}{l}\text { Pernambuco } \\
\text { (Brasil) }\end{array}$ & $02-06-1888$ & 18 & Si : 1909 \\
\hline $9 b$ & 1906 & \begin{tabular}{|l|} 
Ernesto \\
Morelos
\end{tabular} & $\begin{array}{l}\text { Guadalajara } \\
\text { (México) }\end{array}$ & $04-08-1882$ & 24 & Si : 1908 \\
\hline 13 & 1906 & $\begin{array}{l}\text { Rodrigues } \\
\text { de Brito }\end{array}$ & $\begin{array}{l}\text { Campinas } \\
\text { (Brasil) }\end{array}$ & $25-08-1877$ & 29 & No \\
\hline 14 & 1907 & \begin{tabular}{|l} 
Jules \\
Bertrand
\end{tabular} & Iquique (Chile) & $16-10-1888$ & 19 & Si : 1910 \\
\hline 15 & 1907 & \begin{tabular}{|l} 
Ernesto \\
Castro
\end{tabular} & $\begin{array}{l}\text { San José de } \\
\text { Costa Rica }\end{array}$ & $07-02-1891$ & 16 & Si : 1910 \\
\hline 16 & 1907 & $\begin{array}{l}\text { Alberto } \\
\text { Manuel } \\
\text { Blancas }\end{array}$ & Buenos Aires & $01-08-1888$ & 19 & Si : 1911 \\
\hline 17 & 1907 & \begin{tabular}{|l} 
Carlos \\
Sotomayor
\end{tabular} & $\begin{array}{l}\text { Santiago de } \\
\text { Chile }\end{array}$ & $26-10-1889$ & 18 & Si : 1910 \\
\hline 18 & 1908 & $\begin{array}{l}\text { Antoine } \\
\text { Henri } \\
\text { Gibello } \\
\end{array}$ & $\begin{array}{l}\text { Santa Fé } \\
\text { (Argentina) }\end{array}$ & $09-02-1891$ & 17 & Si : 1912 \\
\hline $19 a$ & 1908 & \begin{tabular}{|l} 
Luis María \\
Oyuela
\end{tabular} & Buenos Aires & 02-09-1895 & - & (si) \\
\hline 20 & 1908 & \begin{tabular}{|l|} 
Elysée \\
Scarpa
\end{tabular} & Rio de Janeiro & $01-05-1891$ & 17 & Si : 1912 \\
\hline 21 & 1908 & \begin{tabular}{|l} 
Manuel \\
Amabilis
\end{tabular} & \begin{tabular}{|l} 
Mérida \\
(México)
\end{tabular} & 21-09-1889 & 19 & Si : 1912 \\
\hline $22 \mathrm{a}$ & 1908 & \begin{tabular}{|l|} 
Raoul \\
Castro
\end{tabular} & $\begin{array}{l}\text { San José de } \\
\text { Costa Rica. }\end{array}$ & $20-11-1888$ & - & - \\
\hline 23 & 1908 & José Sosa & Asunción & $28-05-1886$ & 22 & Si: 1912 \\
\hline \multicolumn{7}{|c|}{ Inscritos entre 1910 y 1919} \\
\hline 24 & 1910 & $\begin{array}{l}\text { Ricardo } \\
\text { Oyuela }\end{array}$ & $\begin{array}{l}\text { La Plata } \\
\text { (Argentina) }\end{array}$ & $22-03-1889$ & 21 & Si : 1913 \\
\hline $19 \mathrm{~b}$ & 1910 & $\begin{array}{l}\text { Luis María } \\
\text { Oyuela }\end{array}$ & Buenos Aires & 02-09-1895 & 15 & Si : 1913 \\
\hline 25 & 1910 & $\begin{array}{l}\text { Jules André } \\
\text { Petit }\end{array}$ & Buenos Aires & $17-07-1891$ & 19 & Si : 1913 \\
\hline 26 & 1910 & \begin{tabular}{|l|} 
Adolfo \\
Truan
\end{tabular} & Buenos Aires & $18-02-1892$ & 18 & No \\
\hline 27 & 1910 & $\begin{array}{l}\text { Thomas } \\
\text { Saubidet }\end{array}$ & Buenos Aires & 19-09-1891 & 19 & Si : 1913 \\
\hline 28 & 1911 & $\begin{array}{l}\text { Georges } \\
\text { Bartet }\end{array}$ & Lima & 01-02-1891 & 20 & No \\
\hline 29 & 1911 & Raoul Malet & Rio de Janeiro & $15-04-1892$ & 19 & Si : 1914 \\
\hline 30 & 1911 & \begin{tabular}{|l|} 
Estanislaw \\
Pirovano
\end{tabular} & Buenos Aires & 27-09-1891 & 20 & Si : 1914 \\
\hline 31 & 1911 & \begin{tabular}{|l|} 
Emilio \\
Cádiz
\end{tabular} & $\begin{array}{l}\text { Santiago de } \\
\text { Chile }\end{array}$ & $15-08-1892$ & 19 & No \\
\hline 32 & 1911 & $\begin{array}{l}\text { Fernand } \\
\text { Valdivieso }\end{array}$ & $\begin{array}{l}\text { Santiago de } \\
\text { Chile }\end{array}$ & $06-05-1890$ & 21 & Si : 1914 \\
\hline 33 & 1911 & \begin{tabular}{|l} 
Victor de \\
Vidal \\
\end{tabular} & Rio de Janeiro & $30-07-1889$ & 22 & No \\
\hline 34 & 1912 & Jean Fortini & Buenos Aires & $08-11-1891$ & 21 & No \\
\hline $35 a$ & 1912 & \begin{tabular}{|l|} 
Ascanio \\
Domingues
\end{tabular} & Rio de Janeiro & $08-11-1893$ & - & - \\
\hline 36 & 1912 & \begin{tabular}{|l|} 
Pierre \\
Lajontans
\end{tabular} & Port-au-Prince & $13-06-1896$ & 16 & No \\
\hline 37 & 1912 & $\begin{array}{l}\text { Lucien } \\
\text { Martin }\end{array}$ & $\begin{array}{l}\text { Cap-Haitien } \\
\text { (Haití) }\end{array}$ & $18-10-1893$ & 19 & No \\
\hline 38 & 1912 & $\begin{array}{l}\text { Idelfonse } \\
\text { do Rêgo } \\
\text { Monteiro }\end{array}$ & Recife (Brasil) & $05-03-1896$ & 16 & No \\
\hline 39 & 1913 & \begin{tabular}{|l|} 
Raphaël \\
Auguste \\
Fusoni \\
\end{tabular} & Buenos Aires & $16-02-1896$ & 17 & No \\
\hline $35 b$ & 1913 & \begin{tabular}{|l|} 
Ascanio \\
Domingues
\end{tabular} & Rio de Janeiro & $08-11-1893$ & 20 & No \\
\hline
\end{tabular}




\begin{tabular}{|c|c|c|c|c|c|c|}
\hline 40 & 1913 & \begin{tabular}{|l|} 
Octavio de \\
Gouvea-Freire \\
\end{tabular} & \begin{tabular}{|l} 
Rio Grande do \\
Sul (Brasil) \\
\end{tabular} & 19-03-1892 & 21 & \begin{tabular}{|l|} 
Si : \\
1916 \\
\end{tabular} \\
\hline 41 & 1913 & Luis Siri & Buenos Aires & $30-07-1892$ & 21 & No \\
\hline $22 \mathrm{~b}$ & 1913 & Raoul Castro & $\begin{array}{l}\text { San José de } \\
\text { Costa Rica }\end{array}$ & $20-11-1888$ & 25 & No \\
\hline 42 & 1913 & \begin{tabular}{|l|} 
Caetano \\
Duarte Silva
\end{tabular} & $\begin{array}{l}\text { Belo Horizonte } \\
\text { (Brasil) }\end{array}$ & $14-11-1894$ & 19 & No \\
\hline 43 & 1913 & \begin{tabular}{|l} 
Henry Lizaire \\
\end{tabular} & Port-au-Prince & s.f & ¿? & No \\
\hline 44 & 1913 & $\begin{array}{l}\text { Guillermo } \\
\text { Caimi } \\
\text { Garmendia } \\
\end{array}$ & Buenos Aires & $04-11-1895$ & 18 & No \\
\hline 45 & 1913 & \begin{tabular}{|l} 
Alberto Caimi \\
Garmendia \\
\end{tabular} & Buenos Aires & $06-01-1897$ & 16 & No \\
\hline 46 & 1913 & \begin{tabular}{|l|} 
Samuel \\
Mondragón \\
\end{tabular} & México & $20-01-1895$ & 18 & No \\
\hline 47 & 1913 & $\begin{array}{l}\text { Juan Gabriel } \\
\text { Molina Font }\end{array}$ & \begin{tabular}{|l|} 
Mérida \\
(México)
\end{tabular} & $02-05-1890$ & 23 & No \\
\hline 48 & 1913 & $\begin{array}{l}\text { Cypriano } \\
\text { Lemos }\end{array}$ & Rio de Janeiro & $07-11-1882$ & 31 & No \\
\hline 49 & 1914 & $\begin{array}{l}\begin{array}{l}\text { Pierre Louis } \\
\text { Clément }\end{array} \\
\end{array}$ & \begin{tabular}{|l|}
$\begin{array}{l}\text { Les Gonaives } \\
\text { (Haití) }\end{array}$ \\
\end{tabular} & $27-03-1891$ & 23 & No \\
\hline 50 & 1915 & \begin{tabular}{|l|} 
Ernesto \\
Lemos
\end{tabular} & Buenos Aires & $27-06-1896$ & 19 & No \\
\hline 51 & 1916 & \begin{tabular}{|l} 
Hugo de \\
Azevedo \\
Marques
\end{tabular} & Rio de Janeiro & $19-05-1900$ & 16 & No \\
\hline 52 & 1917 & $\begin{array}{l}\text { Thomaz } \\
\text { Romero } \\
\text { Pereira }\end{array}$ & $\begin{array}{l}\text { Encarnación } \\
\text { (Par.) }\end{array}$ & $04-10-1886$ & 31 & No \\
\hline 53 & 1917 & Raoul Rivero & \begin{tabular}{|l|}
$\begin{array}{l}\text { Saladillo } \\
\text { (Argentina) }\end{array}$ \\
\end{tabular} & $16-08-1890$ & 27 & No \\
\hline 54 & 1918 & $\begin{array}{l}\text { Charles } \\
\text { Malbranche }\end{array}$ & Buenos Aires & 01-09-1901 & 17 & \begin{tabular}{|l|} 
Si : \\
1921 \\
\end{tabular} \\
\hline 55 & 1919 & Porfirio Diaz & México & $17-01-1901$ & 18 & \begin{tabular}{|l|} 
Si : \\
1924 \\
\end{tabular} \\
\hline 56 & 1919 & \begin{tabular}{|l|} 
Eduardo \\
Donoso
\end{tabular} & \begin{tabular}{|l|} 
Santiago de \\
Chile
\end{tabular} & $02-11-1902$ & 17 & No \\
\hline 57 & 1919 & \begin{tabular}{|l|} 
Lorenzo \\
Elizaga
\end{tabular} & México & $11-04-1903$ & 16 & No \\
\hline \multicolumn{7}{|c|}{ Inscritos entre 1920 y 1929} \\
\hline 58 & 1920 & \begin{tabular}{|l|} 
Luis \\
Malaussena
\end{tabular} & Caracas & $27-10-1900$ & 20 & \begin{tabular}{|l|} 
Si : \\
1923 \\
\end{tabular} \\
\hline 59 & 1920 & \begin{tabular}{|l} 
Raoul de \\
Grimaldi \\
\end{tabular} & La Paz & 14-04-1903 & 17 & \\
\hline 60 & 1921 & Luis Estévez & La Habana & $09-05-1899$ & 22 & \begin{tabular}{|l|} 
Si : \\
1924 \\
\end{tabular} \\
\hline 61 & 1921 & \begin{tabular}{|l|} 
Alfredo \\
Gangotena
\end{tabular} & Quito & 19-04-1904 & 17 & No \\
\hline 62 & 1921 & \begin{tabular}{|l} 
Georges \\
Saubidet
\end{tabular} & Buenos Aires & $11-09-1903$ & 18 & \begin{tabular}{|l|} 
Si : \\
1924 \\
\end{tabular} \\
\hline 63 & 1921 & \begin{tabular}{|l} 
Raquel \\
Valenzuela
\end{tabular} & $\begin{array}{l}\text { Santiago de } \\
\text { Chile }\end{array}$ & s.f & $i ?$ & No \\
\hline 64 & 1922 & $\begin{array}{l}\text { Leopoldo } \\
\text { Arosemena } \\
\text { Garland } \\
\end{array}$ & Lima & $19-11-1899$ & 23 & \begin{tabular}{|l} 
Si : \\
1924 \\
\end{tabular} \\
\hline 65 & 1923 & \begin{tabular}{|l} 
Manuel \\
Pizarro
\end{tabular} & México & $20-06-1905$ & 18 & \begin{tabular}{|l|} 
Si : \\
1928 \\
\end{tabular} \\
\hline 66 & 1923 & $\begin{array}{l}\text { Alberto } \\
\text { Marsano }\end{array}$ & Lima & $13-03-1902$ & 21 & No \\
\hline 67 & 1924 & \begin{tabular}{|l|} 
Frédéric \\
Charles \\
Alvarez de \\
Toledo
\end{tabular} & Buenos Aires & $30-10-1906$ & 18 & $\begin{array}{l}\text { Si : } \\
1932\end{array}$ \\
\hline 68 & 1924 & $\begin{array}{l}\text { Manuel da } \\
\text { Costa }\end{array}$ & Rio de Janeiro & $02-04-1900$ & 24 & \begin{tabular}{|l|} 
Si : \\
1929 \\
\end{tabular} \\
\hline 69 & 1924 & \begin{tabular}{|l} 
Edouard \\
Dimet
\end{tabular} & Buenos Aires & $27-02-1907$ & 17 & \begin{tabular}{|l|} 
Si : \\
1929 \\
\end{tabular} \\
\hline 70 & 1924 & $\begin{array}{l}\text { Jean-Pierre } \\
\text { Julio }\end{array}$ & Port-au-Prince & $04-11-1903$ & 21 & No \\
\hline 71 & 1924 & $\begin{array}{l}\text { Auguste } \\
\text { Magloire }\end{array}$ & Port-au-Prince & 06-09-1905 & 19 & No \\
\hline 72 & 1924 & \begin{tabular}{|l|} 
José Negre \\
Zevallos \\
\end{tabular} & Lima & $12-08-1904$ & 20 & No \\
\hline 73 & 1924 & $\begin{array}{l}\text { Joaquín Miro } \\
\text { Quesada }\end{array}$ & Lima & $18-08-1906$ & 18 & No \\
\hline 74 & 1925 & Pierre Maudé & Port-au-Prince & 02-01-1909 & 16 & Si :? \\
\hline 75 & 1925 & \begin{tabular}{|l|}
$\begin{array}{l}\text { Luis Iturralde } \\
\text { Levy }\end{array}$ \\
\end{tabular} & \begin{tabular}{|l|}
$\begin{array}{l}\text { Perreux } \\
\text { (Seine)! }\end{array}$ \\
\end{tabular} & $12-07-1903$ & 22 & \begin{tabular}{|l|} 
Si : \\
1929 \\
\end{tabular} \\
\hline 76 & 1926 & \begin{tabular}{|l|} 
Robert \\
Baussan \\
\end{tabular} & Port-au-Prince & $15-08-1908$ & 18 & \begin{tabular}{|l|} 
Si : \\
1930 \\
\end{tabular} \\
\hline 77 & 1926 & \begin{tabular}{|l} 
Manuel \\
Chacón
\end{tabular} & México & $05-02-1901$ & 25 & No \\
\hline 78 & 1926 & \begin{tabular}{|l} 
Maurice \\
Dabadie
\end{tabular} & $\begin{array}{l}\text { Santiago de } \\
\text { Chile }\end{array}$ & 09-05-1905 & 21 & No \\
\hline 79 & 1926 & \begin{tabular}{|l|} 
Albert \\
Iturralde Levy
\end{tabular} & La Paz & $21-05-1907$ & 19 & \begin{tabular}{|l|} 
Si : \\
1929 \\
\end{tabular} \\
\hline
\end{tabular}




\begin{tabular}{|c|c|c|c|c|c|c|}
\hline 80 & 1926 & \begin{tabular}{|l|} 
Cristóbal \\
Pallares
\end{tabular} & Quito & $25-12-1899$ & 27 & No \\
\hline 81 & 1926 & $\begin{array}{l}\text { César } \\
\text { Martinto }\end{array}$ & Buenos Aires & $16-12-1896$ & 30 & No \\
\hline 82 & 1926 & $\begin{array}{l}\begin{array}{l}\text { Ernesto E. } \\
\text { Paradas }\end{array} \\
\end{array}$ & $\begin{array}{l}\text { Puerto Cabello } \\
\text { (ven.) }\end{array}$ & $26-03-1894$ & 32 & $\begin{array}{l}\mathrm{Si}: \\
1930\end{array}$ \\
\hline 83 & 1926 & Juan Toro & $\begin{array}{l}\text { S. Domingo } \\
\text { (Rep. Domin.) }\end{array}$ & $03-05-1903$ & 20 & $\begin{array}{l}\text { Si : } \\
1929\end{array}$ \\
\hline 84 & 1927 & \begin{tabular}{|l} 
Carlos \\
Martínez
\end{tabular} & $\begin{array}{l}\text { Subachoque } \\
\text { (Colombia) }\end{array}$ & $26-07-1905$ & 22 & No \\
\hline 85 & 1927 & \begin{tabular}{|l|} 
Rafael \\
Olano
\end{tabular} & $\begin{array}{l}\text { Popayán } \\
\text { (Colombia) }\end{array}$ & $26-10-1906$ & 21 & No \\
\hline 86 & 1927 & $\begin{array}{l}\text { Hernando } \\
\text { Uribe } \\
\text { Holguín }\end{array}$ & Bogotá & 20-07-1909 & 18 & No \\
\hline 87 & 1927 & $\begin{array}{l}\text { Jaime Pérez } \\
\text { Lucero }\end{array}$ & $\begin{array}{l}\text { Tucumán } \\
\text { (Argentina) }\end{array}$ & $09-12-1907$ & 20 & No \\
\hline 88 & 1927 & \begin{tabular}{|l|} 
Norberto \\
Palacios
\end{tabular} & Montevideo & $03-08-1907$ & 20 & $\begin{array}{l}\mathrm{Si}: \\
1931 \\
\end{array}$ \\
\hline 89 & 1927 & \begin{tabular}{|l|} 
Carlos \\
Portela \\
\end{tabular} & La Habana & $20-08-1902$ & 25 & No \\
\hline 90 & 1928 & $\begin{array}{l}\text { Norbert } \\
\text { Lynch } \\
\text { Quirno }\end{array}$ & Buenos Aires & $13-08-1911$ & 17 & $\begin{array}{l}\mathrm{Si}: \\
1935\end{array}$ \\
\hline 91 & 1928 & $\begin{array}{l}\text { Julio } \\
\text { Moritan }\end{array}$ & $\begin{array}{l}\text { Paraná } \\
\text { (Argentina) }\end{array}$ & $05-11-1908$ & 20 & No \\
\hline 92 & 1928 & $\begin{array}{l}\text { Carlos de } \\
\text { Olazabal }\end{array}$ & Buenos Aires & 27-03-1911 & 17 & No \\
\hline 93 & 1928 & $\begin{array}{l}\text { Luis Pérez } \\
\text { Quesada }\end{array}$ & Buenos Aires & $20-06-1911$ & 17 & $\begin{array}{l}\mathrm{Si}: \\
1933 \\
\end{array}$ \\
\hline 94 & 1928 & $\begin{array}{l}\text { Fernando } \\
\text { Restrepo }\end{array}$ & $\begin{array}{l}\text { Barranquilla } \\
\text { (Colombia) }\end{array}$ & $11-09-1905$ & 23 & $\begin{array}{l}\mathrm{Si}: \\
1932 \\
\end{array}$ \\
\hline 95 & 1928 & $\begin{array}{l}\text { Juan Luis } \\
\text { Risso }\end{array}$ & Montevideo & $27-03-1911$ & 17 & No \\
\hline 96 & 1928 & $\begin{array}{l}\text { Manuel J. } \\
\text { Sánchez }\end{array}$ & $\begin{array}{l}\text { Riosucio } \\
\text { (Colombia) }\end{array}$ & $18-11-1906$ & 22 & No \\
\hline 97 & 1928 & $\begin{array}{l}\text { Guillermo } \\
\text { Tapia }\end{array}$ & $\begin{array}{l}\text { Guadalajara } \\
\text { (México) }\end{array}$ & $23-02-1905$ & 23 & No \\
\hline 98 & 1928 & $\begin{array}{l}\text { José Luis de } \\
\text { Sucre }\end{array}$ & Caracas & 02-04-1908 & 20 & No \\
\hline 99 & 1929 & José A. Caro & $\begin{array}{l}\text { S. Domingo } \\
\text { (Rep. Domin.) }\end{array}$ & $07-06-1910$ & 19 & $\begin{array}{l}\text { Si : } \\
1933\end{array}$ \\
\hline 100 & 1929 & $\begin{array}{l}\text { Louis } \\
\text { Castillo de la } \\
\text { Parra } \\
\end{array}$ & Bogotá & 13-01-1911 & 18 & No \\
\hline 101 & 1929 & Ernest Ewald & Port-au-Prince & 31-07-1912 & 17 & $\begin{array}{l}\text { Si : } \\
1933\end{array}$ \\
\hline 102 & 1929 & $\begin{array}{l}\text { Ernesto J. } \\
\text { Fabregat }\end{array}$ & México & $30-05-1911$ & 18 & No \\
\hline 103 & 1929 & $\begin{array}{l}\text { Humberto } \\
\text { Ibáñez }\end{array}$ & La Paz & $08-03-1908$ & 21 & No \\
\hline 104 & 1929 & $\begin{array}{l}\text { Luis Carlos } \\
\text { Nava }\end{array}$ & La Paz & $04-11-1909$ & 20 & No \\
\hline 105 & 1929 & \begin{tabular}{|l|} 
Alberto \\
Sánchez
\end{tabular} & $\begin{array}{l}\text { Córdoba } \\
\text { (Argentina) }\end{array}$ & $10-05-1905$ & 24 & No \\
\hline \multicolumn{7}{|c|}{ Inscritos entre 1930 y 1939} \\
\hline 106 & 1930 & \begin{tabular}{|l|} 
Joachim \\
Cueto
\end{tabular} & Buenos Aires & 02-01-1914 & 16 & No \\
\hline 107 & 1930 & $\begin{array}{l}\text { Francisco } \\
\text { Márquez }\end{array}$ & Bogotá & $25-01-1913$ & 17 & No \\
\hline 108 & 1930 & $\begin{array}{l}\text { Ricardo } \\
\text { Orellano }\end{array}$ & $\begin{array}{l}\text { El Tocuyo } \\
\text { (Venezuela) }\end{array}$ & 08-01-1911 & 19 & No \\
\hline 109 & 1930 & $\begin{array}{l}\text { Albert } \\
\text { Orvañanos }\end{array}$ & México & $16-07-1912$ & 18 & No \\
\hline 110 & 1931 & \begin{tabular}{|l|} 
César Luis \\
de Oliveira
\end{tabular} & Buenos Aires & $18-10-1910$ & 21 & $\begin{array}{l}\text { Si : } \\
1934 \\
\end{array}$ \\
\hline 111 & 1931 & $\begin{array}{l}\text { Alphonse } \\
\text { Vergnaud }\end{array}$ & Bogotá & $04-06-1912$ & 19 & No \\
\hline 112 & 1931 & \begin{tabular}{|l|} 
Oscar J. \\
Rivera
\end{tabular} & Talca (Chile) & $25-01-1907$ & 24 & No \\
\hline 113 & 1931 & \begin{tabular}{|l|} 
Horacio \\
Ballvé Cañas
\end{tabular} & Buenos Aires & 30-09-1912 & 19 & $\begin{array}{l}\text { Si : } \\
1935 \\
\end{array}$ \\
\hline 114 & 1932 & $\begin{array}{l}\text { Augustin } \\
\text { Cayeres }\end{array}$ & Puerto Rico & $06-01-1913$ & 19 & No \\
\hline 115 & 1932 & Lucien Price & Port-au-Prince & $24-12-1914$ & 18 & No \\
\hline 116 & 1932 & Miguel Roca & $\begin{array}{l}\text { Córdoba } \\
\text { (Argentina) }\end{array}$ & $10-06-1913$ & 19 & No \\
\hline 117 & 1932 & \begin{tabular}{|l} 
Vitalina \\
Niedermayer
\end{tabular} & $\begin{array}{l}\text { San Francisco } \\
\text { (Argentina) }\end{array}$ & $21-10-1905$ & 27 & No \\
\hline 118 & 1933 & $\begin{array}{l}\text { Julio } \\
\text { Gallardo }\end{array}$ & $\begin{array}{l}\text { Córdoba } \\
\text { (Argentina) }\end{array}$ & $04-10-1910$ & 23 & $\begin{array}{l}\text { Si : } \\
1938\end{array}$ \\
\hline 119 & 1933 & \begin{tabular}{|l|} 
Frederic \\
Noël
\end{tabular} & $\begin{array}{l}\text { Cap-Haitien } \\
\text { (Haiti) }\end{array}$ & $30-01-1910$ & 23 & No \\
\hline 120 & 1933 & $\begin{array}{l}\text { Alberto } \\
\text { Ugalde } \\
\text { Portela } \\
\end{array}$ & Buenos Aires & 07-08-1909 & 24 & $\begin{array}{l}\text { Si : } \\
1937 \\
\end{array}$ \\
\hline 121 & 1934 & \begin{tabular}{|l} 
Facundo \\
Gallardo
\end{tabular} & $\begin{array}{l}\text { Córdoba } \\
\text { (Argentina) }\end{array}$ & $26-10-1911$ & 23 & $\begin{array}{l}\mathrm{Si}: \\
1938 \\
\end{array}$ \\
\hline 122 & 1935 & Paul Manne & Rio de Janeiro & $19-10-1914$ & 21 & No \\
\hline 123 & 1936 & \begin{tabular}{|l|} 
Daniel \\
Frumkin
\end{tabular} & Buenos Aires & 14-03-1918 & 18 & No \\
\hline 124 & 1939 & \begin{tabular}{|l|} 
Rosendo \\
Gallardo
\end{tabular} & $\begin{array}{l}\text { Córdoba } \\
\text { (Argentina) }\end{array}$ & $08-05-1916$ & 23 & No \\
\hline
\end{tabular}

Fuente: Elaboración propia (Archives Bibliothèque ESA).

Nota 1: Se reproduce textualmente la ortografía encontrada en los registros.

Nota 2: Se resaltan sobre fondo gris los 51 diplomados. 
Tabla 4. Latinoamericanos diplomados de la ESA entre 1900 y 1939.

\begin{tabular}{|l|l|l|}
\hline & Nacionalidad & $\mathrm{N}^{\circ}$ estudiantes diplomados \\
\hline 1 & Argentina & 18 \\
\hline 2 & Chile & 7 \\
\hline 3 & Brasil & 5 \\
\hline 4 & México & 4 \\
\hline 5 & Haití & 3 \\
\hline 6 & Bolivia & 3 \\
\hline 7 & Venezuela & 2 \\
\hline 8 & Rep. Dominicana & 2 \\
\hline 9 & Colombia & 1 \\
\hline 10 & Costa Rica & 1 \\
\hline 11 & Cuba & 1 \\
\hline 12 & Paraguay & 1 \\
\hline 13 & Perú & 1 \\
\hline 14 & Puerto Rico & 1 \\
\hline 15 & Uruguay & 1 \\
\hline & TOTAL & $\mathbf{5 1}$ \\
\hline
\end{tabular}

Fuente: Elaboración propia (Archives Bibliothèque ESA).

De igual forma, el periodo de la Segunda Guerra supuso otro paréntesis en este sentido: en efecto, a partir de 1940 no se encuentran registros de inscripción de latinoamericanos en la ESA (en 1939, el último inscrito había sido el argentino Rosendo Gallardo, sin haber obtenido el diploma), y habría que esperar hasta 1948 para encontrar nuevos alumnos provenientes de esta región: se trata del cubano Raul Zequeira, y del haitiano Gérard Roy.

Según las fechas de nacimiento, consignadas en los registros, puede establecerse que, en el caso de los latinoamericanos, el promedio de edad al momento de ingresar a la ESA, fue de 20 años: la mayor parte ingresaban con entre 16 y 20 años cumplidos; y únicamente 11 alumnos tenían 25 años o más al momento de inscribirse (de ellos, sólo 4 tenían 30 años o más).

En este mismo periodo, se presentaron algunos casos de hermanos inscritos de forma simultánea: los argentinos Julio María y Carlos Malbranche; Tomás y Jorge Saubidet; Ricardo y Luis María Oyuela; Guillermo y Alberto Caimi Garmendia; Julio, Facundo, y Rosendo Gallardo; los costarricenses Ernesto y Raúl Castro; y los bolivianos Luis y Alberto Iturralde Levy.

En cuanto al género, resulta evidente la escasa presencia de alumnas latinoamericanas en la ESA, puesto que se registran únicamente 3 inscritas a lo largo de casi medio siglo, y de las cuales ninguna obtuvo el diploma: la primera de ellas fue la chilena Raquel Valenzuela, inscrita en 1921; seguida casi una década después por la argentina Vitalina Niedermayer, inscrita en 1932 (figuras 8 y 9); y dos décadas más tarde se encuentra en los registros a la colombiana Béatrice Chancelle, inscrita en 1951.

Por supuesto, el desempeño académico de los latinoamericanos constituye un tema que puede eventualmente ser estudiado a partir de los documentos disponibles. Así, se encuentran ejemplos de parcours sobresalientes como en el caso del chileno Jules Bertrand, inscrito en 1907 y diplomado en 1910, cuyo trabajo es reconocido en el folio de notas como 
"excellent", calificado en su trabajo final con un puntaje de 416 puntos (sobre 500) y ocupando en aquel último año de estudios el $4^{\circ}$ puesto en un grupo de 31 alumnos (de los cuales se diplomaron 23). Bertrand, quien recibió además el Prix de l'Union Coloniale Française, desarrolló la totalidad de su escolaridad $\left(3^{\circ}, 2^{\circ}\right.$, y $1^{\circ}$ classe $)$ en el seno del atelier de Henri Laffillée (1859-1947), en donde tuvo la oportunidad de compartir con el estudiante costarricense Ernesto Castro, también diplomado en 1910. Bertrand y Castro coincidieron en la ESA con otro latinoamericano, el argentino Alberto Manuel Blancas, cuyo rendimiento en el seno del atelier de Gaston Trélat, fue radicalmente opuesto: aunque finalmente obtuvo su diploma en 1911, Blancas obtuvo resultados académicos muy modestos, teniendo incluso que repetir (redoubler) el tercer año de estudios (1910-1911).

Entre las perspectivas de estudio posibles, especialmente en el caso de los 48 alumnos latinoamericanos que obtuvieron su diploma, sería interesante profundizar en el análisis de aspectos como: la composición por nacionalidades de los ateliers en los cuales estudiaron; el desempeño individual (notas, premios, entre otros); o indagar acerca de los sujets de concours, es decir, de los temas propuestas por la ESA para obtener la promoción anual y el diploma final.

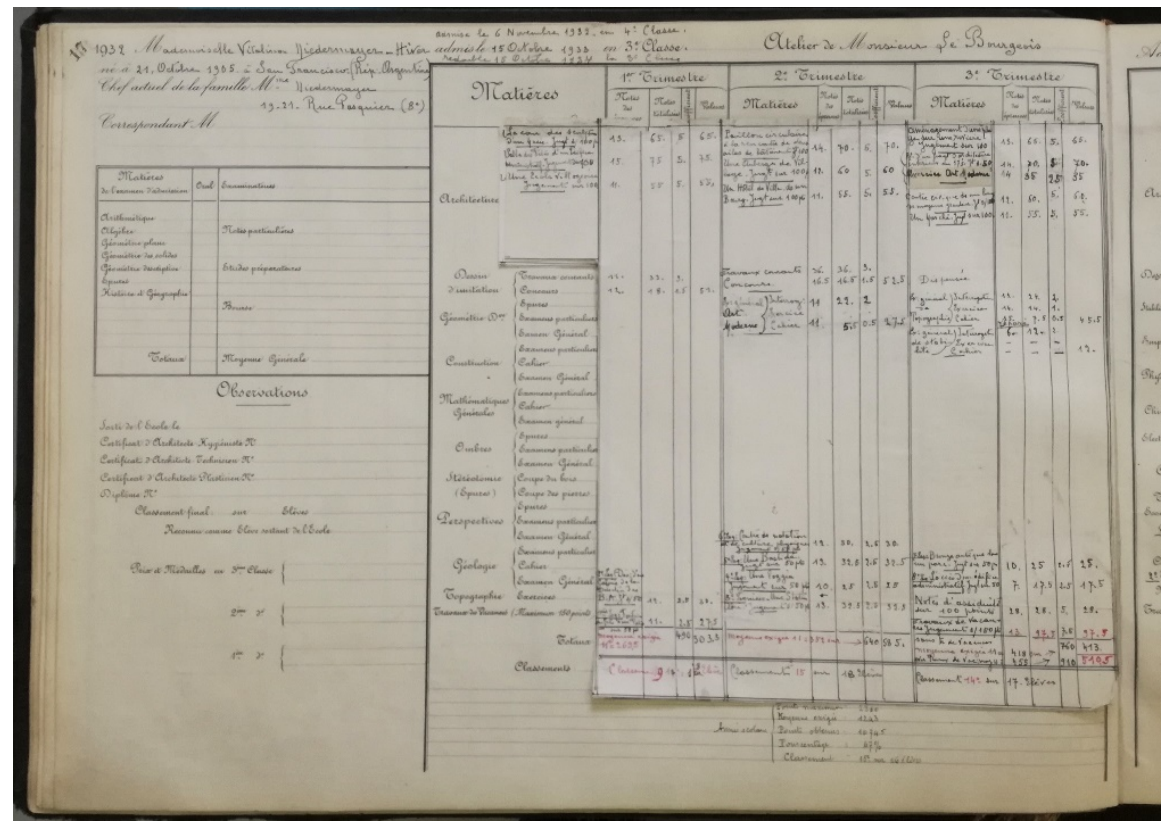

Figura 8. Primera página de notas de la estudiante argentina Vitalina Niedermayer inscrita en 1932. Fuente: Fotografía de Andrés Avila Gómez, junio 2019 (Archives Bibliothèque ESA). 


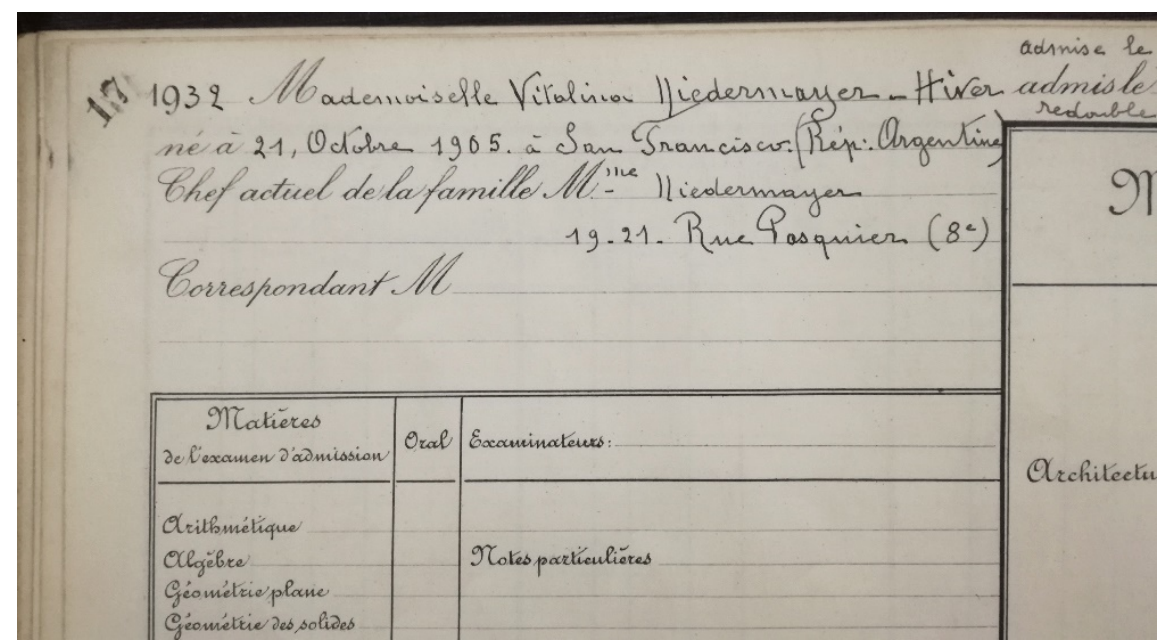

Figura 9. Detalle del encabezado en la primera página de notas de la estudiante argentina Vitalina Niedermayer, quien ingresó a la ESA cuando tenía 27 años.

Fuente: Fotografía de Andrés Avila Gómez, junio 2019 (Archives Bibliothèque ESA).

Tabla 5. Comparativo total de estudiantes diplomados en la ESA (todas las nacionalidades incluidas), y estudiantes latinoamericanos diplomados en la ESA, entre 1900 y 1939.

\begin{tabular}{|c|c|c|c|c|c|}
\hline Año & $\begin{array}{l}\text { Total } \\
\text { diplomados }\end{array}$ & $\begin{array}{l}\text { Latinoamericanos } \\
\text { diplomados }\end{array}$ & Año & $\begin{array}{l}\text { Total } \\
\text { diplomados }\end{array}$ & $\begin{array}{l}\text { Latinoamericanos } \\
\text { diplomados }\end{array}$ \\
\hline 1900 & 19 & 0 & 1920 & 7 & 0 \\
\hline 1901 & 16 & 0 & 1921 & 14 & 1 \\
\hline 1902 & 14 & 0 & 1922 & 22 & 0 \\
\hline 1903 & 14 & 0 & 1923 & 21 & 1 \\
\hline 1904 & 13 & 1 & 1924 & 14 & 4 \\
\hline 1905 & 9 & 1 & 1925 & 14 & 0 \\
\hline 1906 & 12 & 0 & 1926 & 11 & 0 \\
\hline 1907 & 11 & 0 & 1927 & 3 & 0 \\
\hline 1908 & 13 & 2 & 1928 & 21 & 1 \\
\hline 1909 & 21 & 1 & 1929 & 56 & 6 \\
\hline 1910 & 23 & 3 & 1930 & 21 & 2 \\
\hline 1911 & 25 & 1 & 1931 & 28 & 1 \\
\hline 1912 & 20 & 4 & 1932 & 20 & 2 \\
\hline 1913 & 21 & 4 & 1933 & 15 & 3 \\
\hline 1914 & 16 & 3 & 1934 & 9 & 1 \\
\hline 1915 & 8 & 0 & 1935 & 22 & 2 \\
\hline 1916 & 6 & 1 & 1936 & 16 & 0 \\
\hline 1917 & 2 & 0 & 1937 & 17 & 1 \\
\hline 1918 & 2 & 0 & 1938 & 13 & 2 \\
\hline 1919 & 5 & 0 & 1939 & 13 & 0 \\
\hline $\begin{array}{l}\text { TOTAL } \\
1900-1919\end{array}$ & 270 & 21 & $\begin{array}{l}\text { TOTAL } \\
1920-1939\end{array}$ & 357 & 27 \\
\hline
\end{tabular}

Fuente: Elaboración propia (Archives Biblothèque ESA, y Annuaires SADESA).

\section{CONCLUSIONES}

Desde hace aproximadamente dos décadas, los estudios (tesis doctorales, informes oficiales, etc.) y publicaciones francesas que abordan temas relacionados con la historia de la enseñanza de la arquitectura, han puesto en evidencia la relevancia del estudio sobre la presencia de estudiantes extranjeros en las instituciones francesas que ofrecían formaciones en 
arquitectura y/o en urbanismo (especialmente las écoles d'architecture, y algunas écoles d'ingénieurs). Y en esta dirección, se han desarrollado importantes investigaciones entre las cuales sobresalen la de Carmen Popescu sobre alumnos rumanos en la ENSBA; la de Isabelle Gournay sobre alumnos norteamericanos en la ENSBA; y la de Dave Lüthi sobre alumnos suizos en la ENSBA.

En general, el caso francés -aunque puede afirmarse lo mismo sobre el caso alemán, o el italiano- a pesar de la obviedad de su influencia formal en los países latinoamericanos particularmente a través del denominado modelo Beaux-Arts difundido desde el seno de la ENSBA, es aún desconocido por un lectorado hispanófono que ante la ausencia flagrante y lamentable de traducciones, no tiene acceso a los textos que durante el último cuarto de siglo han dado cuenta de juiciosas investigaciones en torno a la enseñanza de la arquitectura en Francia.

Para estudiar a fondo los fenómenos y procesos propios de este tipo de transferencias culturales, y en especial el rol de alumnos y profesionales extranjeros como passeurs de teorías y/o de múltiples savoir-faire a través de una cada vez más rica cultura material, es necesario el aporte y la mirada desde "la otra orilla" ${ }^{[24]}$ : y en el caso latinoamericano, los estudios globales sobre la educación recibida por arquitectos y urbanistas en el extranjero $^{[25]}$, brillan por su ausencia.

Existen algunas excepciones que contrastan con esta evidencia: tal es el caso de los abundantes -y repetitivos- estudios sobre los jóvenes aprendices latinoamericanos en el atelier de Le Corbusier; los cuales representan, sin embargo, un valioso punto de partida para entender este tipo de fenómenos. En resumen, podemos afirmar que en nuestro medio ha corrido más tinta para abordar el tema de la "formación" de los discípulos latinoamericanos de Le Corbusier en su famoso atelier de la rue de Sèvres, que para abordar lo relacionado con la formación de estudiantes latinoamericanos en las écoles d'architecture francesas.

En tal sentido, urge tomar distancia de la irremediable tentación por el estudio de las grandes figuras nacionales de la arquitectura (retomando una y otra vez nombres como Pani, Villanueva, Salmona, etc.), para abrir finalmente un espacio al estudio de personajes considerados como figuras "menores", o que simplemente han sido ignorados por la historiografía latinoamericana de la arquitectura.

En el caso puntual de la École Spéciale d'Architecture, existen diversas perspectivas de estudio, que pueden ser exploradas a partir de las fuentes primarias disponibles; y que al ser complementadas con otro tipo de fuentes - por ejemplo, las revistas de arquitectura, de construcción, y de urbanismo de la época analizada-, pueden arrojar luces sobre cuestiones como la existencia de vínculos profesionales y personales entre ex alumnos de dichas instituciones; o sobre la transferencia de modelos pedagógicos puestos en marcha en las nuevas facultades de arquitectura fundadas durante la primera mitad del siglo XX en países latinoamericanos. 


\section{EPÍLOGO}

En la introducción de su libro Ciudady Arquitectura. Seis generaciones que construyeron la América Latina moderna, la historiadora de la arquitectura Silvia Arango identifica el paso del siglo XIX al XX como una época de "invención de la profesión de arquitectura":

Para comienzos de siglo la arquitectura era una profesión prácticamente desconocida. Los pocos arquitectos de los que se tenía noticia eran una especie extraña y sofisticada que por lo general habían estudiado en el extranjero y no se tenía una definición clara de su trabajo, ni siquiera en aquellos países donde existía alguna tradición de enseñanza de la arquitectura o en los que se había intentado, sin mucho éxito, crear algunas asociaciones gremiales(Arango, 2012: 175).

La posición de Arango, compartida por la mayor parte de los autores latinoamericanos que han construido una historiografía "regional" de la arquitectura durante las últimas cuatro décadas, evidencia serias contradicciones que reflejan a la larga la ausencia de estudios importantes sobre la formación de los arquitectos y urbanistas latinoamericanos en el exterior, y una particular negligencia ante los interrogantes que plantean las problemáticas y procesos derivados de dichos intercambios.

Arango reconoce una tradición de enseñanza de la arquitectura en algunos países, como también la presencia de estudiantes latinoamericanos en instituciones fundamentalmente europeas; pero define al mismo tiempo la arquitectura como una "profesión desconocida" ya entrado el siglo XX. Esto se explica quizás por la pulsión de la autora $-\mathrm{y}$ de los adeptos de esta "escuela negacionista" de las transferencias, tan presente en la historiografía latinoamericana- de cuestionar radicalmente "la noción de influencia" (Arango, 2012: 14), puesto que según su interpretación:

[...] las formas arquitectónicas y urbanas que se hicieron en América Latina en la época moderna no vienen de formas generadas en otra parte, que copiamos, adaptamos o adoptamos, sino del hecho de compartir y debatir ideas generales que produjeron, creativamente, alternativas arquitectónicas en suelo latinoamericano.

Aunque es claro que en su discurso, la autora pretende distanciarse deliberadamente de aquellos otros (Pevsner, Giedion, Zevi, Benévolo, Banham, Collins, Tafuri, etc.) "con pretensiones universales pero con un marcado eurocentrismo" (Arango, 2016: 13), parece olvidar en su declaración de intenciones que la "invención de la profesión de arquitectura" ya llevaba varios siglos de evolución en países como Francia (la Académie Royale d'Architecture fue fundada en 1671), o España (la Real Academia de San Fernando fue fundada en 1752) y que fueron precisamente experimentos europeos (la École des Beaux-Arts al iniciar el siglo XIX, la Bauhaus a comienzos del siglo XX), los que sirvieron como modelos para la creación de los primeros programas y de las primeras facultades de arquitectura en territorio latinoamericano inicialmente en algunos países durante la segunda mitad del siglo XIX, ${ }^{[26]}$ y más tarde en otros países durante la primera mitad del siglo XX. 
Si para la autora, el énfasis de una nueva historia vista desde América Latina "[...] no está depositado en los objetos arquitectónicos sino en sus actores" (Arango, 2012: 14), no se comprende la razón por la cual el tema de la formación de aquellos actores (escolaridad, lecturas, viajes, etc.) sea relegado en el relato histórico a un plano absolutamente secundario y casi anecdótico.

\section{FUENTES DE CONSULTA}

Arango, S. (2012), Ciudad y arquitectura. Seis generaciones que construyeron la América Latina moderna, Fondo de Cultura Económica, México.

Ávila Gómez, A. (2019a), "Estudiantes colombianos en el Institut d'Urbanisme de l'Université de Paris durante el período de entreguerras", Dearq, núm. 25, pp. 178-189. Disponible en https://revistas.uniandes.edu.co/ doi/pdf/10.18389/dearq25.2019.09.

Ávila Gómez, A. (2019b), "Estudiantes latinoamericanos en el Institut d'Urbanisme de l'Université de Paris, 1926-1941", Revista de Arquitectura, vol. 21, núm. 2, pp. 44-56. Disponible en https://editorial.ucatolica.edu.co/ojsucatolica/ revistas_ucatolica/index.php/RevArq/article/view/2154.

Ávila Gómez, A. y Quintana Guerrero, I. (2018), "Formation en architecture et urbanisme de jeunes colombiens à Paris, 1927-1954". Cahier HEnsA20 - Histoire de l'Enseignement de l'Architecture au 20e siècle, núm. 4, pp. 15-20. https://chmcc.hypotheses.org/4258

Botti, G. (2017), "Geographies for Another History: Mapping the International Education of Architects from Colombia, 1930-1970". Architectural Histories, 5 (1), https://journal.eahn.org/articles/10.5334/ah.230/

Dumont, M-J. (1989), La S.A.D.G., histoire d'une société d'architectes. Première partie : 1877-1939. Société Française des Architectes, Paris. https://sfarchi.org/wp-content/uploads/pdf/SADGtome1.pdf

Jiménez Madera, L. (2015), "Los arquitectos latinoamericanos en la École des Beaux-Arts de París en el siglo XIX", Revista de Arquitectura, vol. 17, núm. 1, pp. 73-84. Disponible en https://editorial.ucatolica.edu.co/ ojsucatolica/revistas_ucatolica/index.php/RevArq/article/view/87.

Lambert, G. (2014), "La pédagogie de l'atelier dans l'enseignement de l'architecture en France aux XIXe et XXe siècles : une approche culturelle et matérielle", Perspective, (1), pp. 129-136. Disponible en https:// journals.openedition.org/perspective/ 4412

Lucan, J. (2017), Composition, non-composition. Architecture et théories, XIXe-XXe siècles. Presses Polytechniques et Universitaires Romandes, Lausanne.

Matus Carrasco, D. (2018), "Diplômes et diplômés en urbanisme, Paris 1919-1969". Tesis doctoral, Université Paris Nanterre, París.

Moulin, R., et al. (dir.) (1973), Les architectes : métamorphose d'une profession libérale. Calmann-Lévy, París.

Peliowski, A. (2018), "Lo bello o lo útil. Ideologías en disputa en torno a la creación del primer curso universitario de arquitectura en Chile, 1848-1953". Historia, núm. 51, vol. 2, pp. 485-515. 
Seitz, F. (1995), L’École Spéciale d'Architecture, 1865-1930. Une entreprise d'idée. Picard, París.

Seitz, F. (1993), L'enseignement de l'architecture en France au XIXe siècle. Les Cahiers du Centre de Recherche Historiques, núm. 11. Disponible en https://journals.openedition.org/ccrh/2768

Société des Architectes Diplômés de l'École Spéciale d'Architecture. Annuaire SADESA 1998-1999, París.

Vacher, H. (2016), "L'École Spéciale des Travaux Publics et la formation des ingénieurs - architectes au premier vingtième siècle", Cahier HEnsA20 - Histoire de l'Enseignement de l'Architecture au 20e siècle, núm. 2, pp. 37-44. Disponible en https://chmcc.hypotheses.org/3129.

\section{Notas}

[1]Agradecemos la colaboración irrestricta y permanente de Anne Chaise, encargada de la Bibliothèque de la École Spéciale d'Architecture.

[2] Ver los tres artículos más recientes (Ávila Gómez, 2018, 2019a, 2019b).

[3] Sobre los latinoamericanos formados en la ENSBA en el siglo XIX, ver el artículo de Jiménez Madera: «Los arquitectos latinoamericanos en la École des Beaux-Arts de París en el siglo XIX». Revista de Arquitectura, vol. 17, n 1 (2015).

[4]Trélat, L'Enseignement des beaux-arts, Paris, 1864, p. 14 ; Trélat, L'École centrale d'architecture, Paris, 1864, p. 26.

[5] «Oponiéndose a toda "teoría de lo hermoso invariable, de lo bello circunscrito en los tipos, de lo bello encontrado de una vez por todas en tierras privilegiadas, de lo bello originado en Grecia y reeditado en Roma", Trélat buscó desarrollar una enseñanza más racional, a semejanza de aquella impartida a los ingenieros civiles, sin por ello abandonar la lógica del sistema de ateliers: "El atelier, -es necesario repetirlo-, constituye el centro en torno al cual gravita todo el sistema de enseñanza.» [Traducido por Andrés Ávila Gómez].

[6]Ver el listado con notas biográficas establecido por Seitz, bajo el título «Itinéraires de 100 architectes diplomés de l'École Centrale puis Spéciale d'Architecture» (Seitz, 1995: 146-170).

[7] «Y sucedió lo que debía suceder! Un señor, arquitecto, y padre de uno de los alumnos de Mallet-Stevens, apareció delante del Sr. [Gaston] Trélat, director de la ESA. El arquitecto blandía su paraguas mientras gritaba: «Mi opinión puede importarle un comino; justed quiere enloquecer a mi hijo! ¡Usted amarga su existencia, lo envilece! Mi hijo no tendrá jamás encargos como arquitecto» El Sr. director de la ESA se puso a temblar de verdad, y luego alcanzó a Mallet-Stevens para expresarle que tal situación no podía continuar, que era necesario revertir todo aquello, regresando a las tradiciones de su honorable escuela. ¡Santo Dios! El propio Sr. Trélat había hecho allí una carrera, por lo demás modesta. Siempre orgulloso, Mallet-Stevens se marchó. Mientras tanto, los jóvenes y entusiastas alumnos de arquitectura de la ESA terminarán sus estudios entre la lectura de Vignole y la contemplación del Grand Palais [...]» [Traducido por Andrés Ávila Gómez].

[8]La violenta crítica de Viollet-le-Duc a la ENSBA fue expuesta en su célebre obra Entretiens de l'architecture, encontrando continuidad en su alumno y discípulo Anatole de Baudot. Tres puntos centrales eran atacados por Viollet-le-Duc: la dictadura ejercida por la ENSBA en materia de doctrina arquitectónica; las evidentes lagunas presentes en la estructura de la formación impartida allí; y la consecuente falta de preparación de los arquitectos egresados de la ENSBA para ejercer la profesión (Seitz, 1993: 5). 
[9]Aunque los primeros diplomas conferidos por la ENSBA datan de 1869 (apenas 4 laureados), fue a partir de 1914 cuando el gobierno concedió una base oficial al mismo agregando el título de "architecte diplomé pour le gouvernement" que da origen a las iniciales DPLG (Dumont, 1989: 25).

[10]Sobre la difusión del modelo pedagógico basado en la enseñanza en el atelier, ver especialmente el texto sintético de Lambert (2014) [Existe una traducción al español (2017)].

\section{[11]Citado por Seitz (1995: 89 y 91).}

$[12] \ll$ Es en el atelier donde se realizan los trabajos fundamentales que constituyen el ejercicio permanente de la arquitectura, despertando así al artista, gracias a una lenta asimilación de los procedimientos de expresión reservados al arquitecto: es allí en donde se consolidan los estudios progresivos conocidos bajo el nombre de copias, planos, proyectos (borradores o presentaciones), concursos, etc.; según el nivel de progreso del alumno. En el atelier, el alumno es libre: allí distribuye y utiliza su tiempo como lo desea, siempre con el propósito de satisfacer de la mejor manera las exigencias derivadas de la educación que recibe; pero también aprovechando al máximo los consejos que regularmente da el arquitecto director del atelier, y beneficiándose cuando le es posible de los textos disponibles en la biblioteca de la institución » [Traducido por Andrés Ávila Gómez].

[13]Concurso de Técnica: Certificado de arquitecto técnico.

[14]Concurso de Plástica: Certificado de arquitecto plástico.

[15]Concurso de Salubridad: Certificado de arquitecto higienista.

[16]Antiguo alumno de la ESA, y ganador en 1902 del prestigioso Grand Prix de Rome otorgado por la ENSBA.

[17]Paralelamente, la École Nationale Supérieure des Arts Décoratifs (ENSAD), que como escuela pública había creado un diploma de Arquitectura en 1922 (reconocido oficialmente en 1931, expedido por el ministro de la Instruction publique et des BeauxArts), tomaba también la decisión de incluir en sus filas como docentes, a arquitectos del prestigio de Robert Mallet-Stevens y Georges-Henri Pingusson (1894-1978).

[18]Cabe recordar que ya en 1909 se diplomaban los primeros alumnos de la formación específica en arquitectura implementada por la École Spéciale des Travaux Publics du Bâtiment et de l'Industrie (ESTPBI, también denominada ESTP). Para 1939, los diplomas de "ingénieur-architecte" otorgados por esta institución, tienen ya un prestigio similar al de aquellos dados en la ENSBA, la ESA y la ENSAD (Vacher, 2016).

[19]El estudio de Seitz, y algunas otras fuentes, mencionan erróneamente al arquitecto suizo Jean-Édouard Jéquier como el padre de Jules-Émile.

[20]Matricula $\mathrm{n}^{\circ} 3949$.

[21] Actualmente, la arquitecta Yolanda Muñoz desarrolla en el seno de la Escuela doctoral de Arquitectura, de la Pontificia Universidad Católica de Chile, una tesis doctoral acerca de la vida y obra de Jules-Émile Jéquier.

[22] Sobre los latinoamericanos en el IUUP, ver los artículos de Ávila Gómez (2019a y 2019b).

[23] Matricula $\mathrm{n}^{\circ} 7289$.

[24]Vale la pena resaltar, por ejemplo, la tesis doctoral de Daniel Matus Carrasco, titulada: «Diplômes et diplômés en urbanisme, Paris 1919-1969», sustentada en 2018.

[25] Sobre el caso colombiano, por ejemplo, Giaime Botti presenta en un artículo publicado en 2017, un panorama global sobre la formación recibida entre 1920 y 
1970 por aproximadamente 200 arquitectos colombianos. Sin embargo, al apoyarse principalmente en fuentes secundarias, los resultados expuestos pueden ser bastante inexactos: en el caso francés no se menciona a ninguno de los 8 colombianos que estudiaron en la ESA; y sobre aquellos que estudiaron en la ENSBA y en el IUUP se presentan datos incompletos (en el caso del IUUP, por ejemplo, se diplomaron 6 colombianos entre 1930 y 1967).

[26]En la Universidad de Chile, por ejemplo, se creó en 1849 el primer curso de arquitectura: y para ello, el gobierno chileno contrató entonces al arquitecto francés Claude-François Brunet Debaines (1799-1855). Sobre este episodio en particular, ver el texto de Amari Peliowski titulado "Lo bello o lo útil. Ideologías en disputa en torno a la creación del primer curso universitario de arquitectura en Chile, 1848-1953" (2018). 